\title{
IgA and IgG antibodies in SARS-CoV-2 vaccinated health workers by a homemade ELISA diagnostic test
}

\author{
Tagliaferro Luigi ${ }^{1}$, Menegazzi Paola ${ }^{1}$, Cossa Luca Giulio², Maffia Michele ${ }^{2}$
}

\begin{abstract}
The SARS-CoV-2 pandemic has accelerated vaccine development and testing, an important step in its eradication. Health workers were included among the first subjects to be vaccinated, therefore it was necessary to check their immunological status after the first and second dose of vaccine.

Serum samples belonging to 24 health workers undergoing vaccination for SARS-CoV-2 (Pfizer-BioNTech) were analysed: for 2 of them it was also possible to obtain a serum sample prior to the first dose of vaccine (zero time); antibody dosing was performed in 18 out of 24 after the first and second vaccination dose; in the remaining 6 healthcare workers a serum sample was obtained only after the second dose. In each serum sample, IgA and IgG antibodies to "Spike Receptor Binding Domain" (RBD) and "Nucleocapsid" (N) proteins were searched by ELISA test.

Except for the two subjects for whom it was possible to have a serum sample before vaccination, the others were collected on day 18 from the first dose of vaccine and on day 8 from the second dose.

Serum samples collected after the first dose of vaccine showed reactivity to anti-RBD IgG in 11 out of 18 healthcare workers and to IgA in 2 subjects. After the second dose all 24 samples showed the presence of anti-S IgG, while 5 revealed a reactivity for anti-S IgA. Three samples showed reactivity towards anti-N IgG.

The ELISA test has shown all its effectiveness in controlling post vaccine immunity and in discriminating natural immunity from vaccine induced immunity.
\end{abstract}

Keywords: COVID-19; ELISA; SARS-CoV-2; IgG; IgA

${ }^{1}$ Molecular Biology Unit, "S. Caterina Novella"

Hospital, Galatina (LE), Italy.

${ }^{2}$ Department of Biological and Environmental

Sciences and Technologies, University of Salento,

73100 Lecce, Italy.

${ }^{*}$ Corresponding Author:

Maffia M., Department of Biological and

Environmental Sciences and Technologies, University

of Salento, 73100 Lecce, Italy. Email: michele.maffia@ unisalento.it

DOI: 10.2478/ebtj-2021-0016

(C) 2021 Authors. This work was licensed under the

Creative Commons Attribution-NonCommercial-

NoDerivs 4.0 License.

\section{Introduction}

In the late 2019, a cluster of patients with atypical pneumonia was diagnosed in $\mathrm{Wu}$ han, China (1). The etiological agent was isolated and its genome sequenced, which was found to be an emergent zoonotic virus, member of the Coronaviridae family, genetically related, but not a direct descendent, of another emergent coronavirus, the severe acute respiratory syndrome coronavirus (SARS-CoV) $(1,2)$. The novel emergent virus named SARS-CoV-2 exhibits a rapid human-to-human spreading via the respiratory tract, and is responsible of a syndrome known as Coronavirus disease of 2019 (COVID-19) (3).

The SARS-CoV-2 coronavirus is a viral strain belonging to the subgenus Sarbecovirus, of the subfamily of coronaviruses (Orthocoronavirinae), responsible for diseases ranging from the common cold to more serious diseases such as Middle Eastern respiratory syndrome (MERS) and severe acute respiratory syndrome (SARS). Coronaviruses are a large family of viruses, but only six (229E, NL63, OC43, HKU1, MERS-CoV, SARS-CoV) were previously known for their ability to infect humans; therefore SARS-CoV-2 is the seventh.

The substantial difference from the previous ones is the incubation period, which goes from 2 to 14 days during which it does not cause any symptoms. The World Health Organization (WHO) estimates that the net reproduction rate of person-to-person trans- 
mission of the virus is between 1.4 and 3.8. This value indicates the number of other people to whom a newly infected patient can transmit the disease, thus qualifying the new SARS-CoV-2 as infectious as the 2002 SARS-CoV. During an update conference on January 29, 2020, the World Health Organization makes it known that the method of transmission is not only direct, but also indirect by contact.

The SARS-CoV-2 genome has $89 \%$ nucleotides identical to those of SARS-like-CoVZXC21 (common in bats) and $82 \%$ identical to those of SARS-CoV; however, only $40 \%$ of amino acids coincide with those of SARS-related coronaviruses.

It is not yet known how the virus may have moved from cold-blooded to warm-blooded guests. On January 22, 2020, the Journal of Medical Virology published a report with genomic analysis indicating that snakes in the Wuhan area are "among wildlife, the most likely reservoir" for the virus, but more research is needed.

Since its discovery, COVID-19 has become a pandemic outbreak with more than 129 million people tested positive for the virus, which has resulted in more than 2.8 million deaths around the world by early April 2021(4). According to information displayed on the World Health Organization (WHO) COVID-19 Dashboard, even in countries where the number of daily cases has dropped (e.g., China, Australia), it is not possible to say that the virus has disappeared since active cases are still reported daily. For instance, in regions such as Europe and North America, and coinciding with the autumn and winter seasons, a dramatic increase in the number of reported cases has forced some countries (e.g., Italy, France, Germany) to tighten sanitary restrictions. The SARS-CoV-2 infection clinical manifestation is complex, varying from asymptomatic or mild symptoms in more than $80 \%$ of carrier individuals, to severe respiratory and systemic distress, including obstructive pneumonia, hypoxia, pro-inflammatory cytokine storm, coagulopathies, neurocardiovascular disorders, and death. The mortality rate range from 1 to $3 \%$ among symptomatic patients, depending on medical infrastructure and early medical intervention (5-10).Disease severity variations among infected patients are associated to the individual susceptibility, mainly determined by the density of the viral cellular receptor, angiotensin-converting enzyme II (ACE2). This is significantly higher in individuals older than 50 years, or patients with chronic neurocardiovascular comorbidities, such as diabetes mellitus, hypertension, and obesity $(6,11)$.

To this day, neither specific prophylactic nor therapeutic treatment, including antiviral drugs or molecules to reduce severe symptoms, are available, given the novelty of SARS$\mathrm{CoV}-2$, and the lack of regulatory-approved treatments for other coronavirus and related diseases. Consequently, and given the need for rapid development of effective chemotherapeutic compounds for COVID-19 treatment, the current approach has been the repurposing of existing drugs used in unrelated pathologies, especially those that lack significant side effects in the past.

Another strategy for the systematic fight against the novel
COVID-19 pandemic resides in the prevention of infection through immunization of uninfected individuals with effective vaccine to induce protective anti-SARS-CoV-2 immuneresponses and long-lasting memory.

There is little information regarding the mechanisms and role of the humoral response in SARS-CoV-2 infection. Such information is very important for pandemic risk assessment, for designing the strategies based on serological tests, for predicting vaccine efficacy and for patient monitoring. In particular, a very important aspect is to distinguish the antibody kinetics and dynamics in patients vaccinated from those naturally infected.

Serological tests represent a valuable tool for monitoring viral spread in the population (12).

Moreover, thanks to serological tests it is possible to diagnose previous infections in subjects with levels of viral RNA undetectable by RT-PCR for epidemiological purposes (13). Current available SARS-CoV-2 serological tests do not always allow for a simultaneous detection of different types of antibodies to different antigens reliably, and this it may hinder population studies for clinical or epidemiological purposes (14). In contrast, serological tests based on immunoenzymatic techniques (ELISA) for the assay of antibodies against the Spike Soluble Ectodomain (Spike) and its binding domain of the highly immunogenic receptor (RBD), or against the Nucleocapsid $(\mathrm{N})$ protein, provide promising results in terms of accuracy and reproducibility (15). These ELISA tests made it possible to demonstrate that neutralizing antibodies (nAb) against various viral antigens may decrease after 20-30 days from the onset of symptoms and that the extent of the $\mathrm{nAb}$ response may be associated with disease severity in COVID-19 patients (16).

Therefore, it is useful to have some diagnostic systems capable of monitoring the effectiveness of a vaccine and at the same time being able to distinguish an immunity from natural infection from that following vaccination. So, in this study, a cohort of SARS-CoV-2 vaccinated health workers were subjected to the detection of anti-SARS-CoV-2 IgA and IgG to "RBD" (S) and " $\mathrm{N}$ " viral proteins. The antibody levels were analysed according to the test operating protocol.

\section{Materials and Methods \\ Study Design and Participants}

This study was part of the "COVID-19-SIEROS" project, promoted by the Local Health Authority (ASL) and the Diffuse Laboratory of Interdisciplinary Research applied to Medicine (DReAM).

Immediately after 27 December 2020 (the first day for CoViD vaccination in Italy), the health workers of the "Santa Caterina Novella" hospital in Galatina (LE), Italy, were called to receive the first dose of Pfizer vaccine mRNABNT162b2 (Comirnaty), on different dates, with a second dose however on 21 st day after the first one.

A total of 24 health workers were subjected to post-vaccine serology. Thus, it was possible to collect serum samples for 18 of these already from the first dose, for all on the 18th day from 
the first inoculation of the mRNA vaccine. All samples were collected between December 2020 and January 2021, stored at $-20{ }^{\circ} \mathrm{C}$ until ELISA test performing. For 6 of the 24 recruited subjects, it was possible to obtain the serum sample only after the second dose; for all enrolled subjects, blood sampling after the second dose was performed on the eighth day. For two of them it was possible to analyse a serum sample taken before vaccination (time zero).

All involved subjects were repeatedly negative for SARSCoV-2 molecular test prior to vaccination.

\section{ELISA Serological Assay: detection of SARS-CoV-2 "S" and "N" IgA/IgG}

The serological assay was designed to separately identify antibodies to S-RBD and $\mathrm{N}$ proteins. Thus each 96-well ELISA plate was coated separately overnight at $4{ }^{\circ} \mathrm{C}$ with $2 \mu \mathrm{g} / \mathrm{mL}$ SARS-CoV-2 RBD ectodomain Spike protein and with $2 \mu \mathrm{g} / \mathrm{ml}$ of protein $\mathrm{N}$, kindly provided by the European Institute of Oncology (IEO, Milan). Blocking was performed by 1 hour incubation at room temperature (RT) with Phosphate Buffer Saline and Tween 20 (PBS-T) + 3\% Bovine Serum Albumin (BSA) (weight/volume).

Health workers sera were analysed according to homemade ELISA protocol (17). Serum samples were diluted 1:200 in PBS with $1 \%$ of BSA; $50 \mathrm{ml}$ of diluted serum were incubated at RT for 90 minutes and revealed after a 60 min incubation at RT with secondary anti-human-IgG and IgA antibodies, conjugated to HRP. Plates are read at a $450 \mathrm{~nm}$ wavelength. As suggested in the adopted protocol a cut-off of $0.277450 \mathrm{~nm}$ O.D. was utilized for anti-RBD IgG, 0.290 O.D. for anti-RBD IgA, 0.326 O.D. for anti-N IgG and 0.381 O.D. for anti-N IgA.

\section{Ethical Aspects}

The study was approved by the Ethical Committee of the Lecce Local Health Authority (ASL/LE) on April 21, 2020, with deliberation n. 45. All data were collected and analysed confidentially in accordance with Italian laws (Legislative Decree n. 196 of 30 June 2003, and subsequent additions), for research purposes.

\section{Results}

To evaluate the efficacy of SARS-CoV-2 vaccine, altogether a total of 42 serum samples were tested for IgA and $\operatorname{IgG}$ antibodies to viral Spike Receptor Binding Protein (S-RBD) and nucleoprotein $(\mathrm{N})$ in health workers, vaccinated with Pfizer vaccine mRNABNT162b2 (Comirnaty). Characteristics of the analysed health workers sera are summarized in table 1 and figure 1 .

Prior to vaccination, all healthcare professionals involved in this study had repeatedly tested negative for SARS-CoV-2 by real time RT-PCR.

\section{Time zero samples}

In the two samples taken at time zero there were no $\operatorname{IgG}$ and IgA towards the "S" protein, nor anti "N" IgA, while in one there were anti "N" IgG, also confirmed in the assay after the first dose of vaccine, but not after the second one (health worker AM, tab. 2).

\section{First dose samples}

Of the 18 serum samples collected on day 18 from the first vaccine dose, 8 had discrete IgG anti-S (RBD) levels, 3 were borderline and 7 were negative. Anti-S (RBD) IgA was found in 2 patients, flanked by the presence of IgG. Surprisingly, antibodies to the SARS-CoV-2 nucleoprotein "N" were found in 3 out of 18 patients, including health worker AM at zero time (borderline, see table 2), while anti-N IgA was not found in any of them (table 1 and figure 1D).

\section{Second dose samples}

As expected, all 24 serum samples collected on day eight from the second vaccine dose, including 18 of which were discussed earlier, had good anti-S IgG (RBD) levels (figure 2A). Five of these, including the two positives at the first dose, showed the production of anti-S (RBD) IgA, one of which was at borderline levels (table 1 and figure 1). Interestingly, health worker AM, previously positive for anti-N IgG, became negative after the second dose of vaccine. Health worker ARB, on the other hand, who tested negative for anti-N IgG after the first dose, saw these antibodies positivize after the second dose. Health worker PT remained positive for anti-N IgG even after the second dose of vaccine (table 2 ).

No serum samples showed anti-N IgA after the second vaccine dose (figure 2B).

\section{Discussion}

The cohort of 24 health workers vaccinated with Pfizer $m R N$ ABNT162b2 (Comirnaty) has undoubtedly constituted a useful platform for testing the performance of a practical, flexible and easy-to-use enzyme immunoassay system. The separate search for specific antibodies against the main viral components, including the Spike protein (Receptor Binding Domain, also produced by the Pfizer vaccine) and the nucleoprotein $\mathrm{N}$, allowed to evaluate the efficacy of the vaccine, distinguishing the antibodies produced by it from those of any natural infections.

The enzyme immunoassay (ELISA), object of this study, proved to be easy to perform in any diagnostic laboratory and with a very low cost/test. In fact, it only needs a spectrophotometer for microplates, easily obtainable even in countries with scarce economic resources.

After the first dose of vaccine only slightly more than half of the vaccinated subjects (55\%) showed the production of IgG antibodies on the 18th day of inoculation, of which 3 were mild. Only after the second dose was there a massive production of immunoglobulin G, regardless of gender and age. The presence of IgA was found in 2 subjects after the first dose of vaccine and in 5 subjects after the second dose: they reported the presence of symptoms after vaccination inoculation.

In six cases the presence of anti-SARS-CoV-2 nucleoprotein (N) IgG was found: as described in tables 1 and 2 health worker 
Table 1. Characterization of the health workers' serum.

\begin{tabular}{|c|c|c|c|c|c|}
\hline & \multirow{2}{*}{ Results } & \multicolumn{2}{|c|}{ Anti-S } & \multicolumn{2}{|c|}{ Anti-N } \\
\hline & & IgG & $\operatorname{Ig} A$ & IgG & $\operatorname{Ig} A$ \\
\hline \multirow{4}{*}{$1^{\text {st }}$ dose } & Negative & 7 & 16 & 15 & 18 \\
\hline & Borderline & 3 & 0 & 1 & 0 \\
\hline & Positive & 8 & 2 & 2 & 0 \\
\hline & total & 18 & 18 & 18 & 18 \\
\hline \multirow{4}{*}{$2^{\text {nd }}$ dose } & Negative & 0 & 19 & 22 & 24 \\
\hline & Borderline & 0 & 1 & 0 & 0 \\
\hline & Positive & 24 & 4 & 2 & 0 \\
\hline & total & 24 & 24 & 24 & 24 \\
\hline \multirow{4}{*}{ Zero time } & Negative & 2 & 2 & 1 & 2 \\
\hline & Borderline & 0 & 0 & 0 & 0 \\
\hline & Positive & 0 & 0 & 1 & 0 \\
\hline & total & 2 & 2 & 2 & 2 \\
\hline
\end{tabular}

А

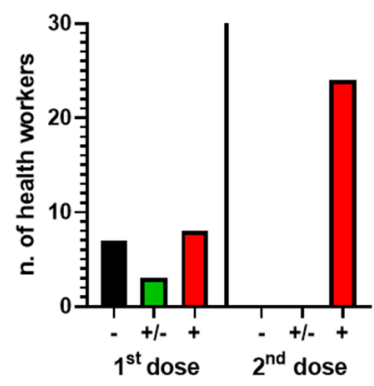

c

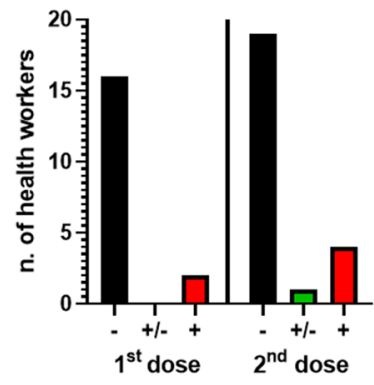

$B$

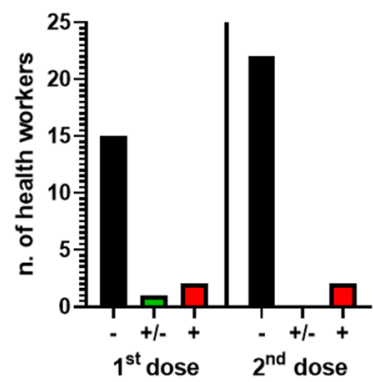

D

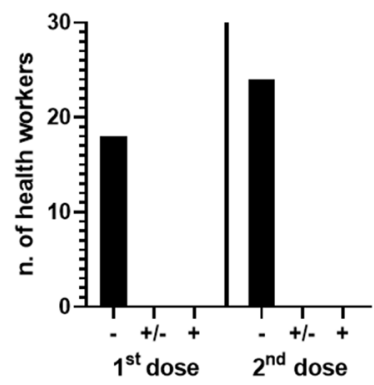

Figure 1. Histograms show the characterization of serum of health workers: (A) anti-RBD ectodomain Spike protein IgG; (B) anti-nucleocapside protein IgG; (C) anti-RBD ectodomain Spike protein IgA; (D) anti-nucleocapside protein IgA.

AM already had these antibodies at time zero and to a weak extent even after the first dose, then it becomes negativized later. Conversely, health worker PT retained anti-N IgG even after the second dose, while DS and ARB showed anti-N IgG only after the second and after the first dose, respectively. Healthcare workers PT and DS work in an infectious disease hospital department.

In this context, the presence of anti-N IgG in subjects vaccinated against SARS-CoV-2 and negative to the molecular swab can be explained as a consequence of seasonal reinfections by other Coronaviruses (with a kinetics of 6-12 months) or with a non-specific cross-reactivity (17).

\section{Conclusion}

In controlling the current pandemic and, in any case, to comply with a correct pre- and post-vaccination laboratory serological diagnosis, the use of a fast, practical and economical device is highly desirable. The ELISA system able to identify the pres 
Table 2. IgG and IgA levels in the serum of each health worker who underwent serological test.

\begin{tabular}{|c|c|c|c|c|c|c|c|c|c|c|c|}
\hline & & & & \multicolumn{4}{|c|}{ Anti-S (RBD) antibody } & \multicolumn{4}{|c|}{ Anti-N antibody } \\
\hline & & & & \multicolumn{2}{|c|}{$\begin{array}{c}\text { IgG } \\
\text { Cut off: } 0.277 \text { OD }\end{array}$} & \multicolumn{2}{|c|}{$\begin{array}{c}\text { IgA } \\
\text { Cut off: } 0.290 \text { OD }\end{array}$} & \multicolumn{2}{|c|}{$\begin{array}{c}\text { IgG } \\
\text { Cut off: } 0.326 \text { OD }\end{array}$} & \multicolumn{2}{|c|}{$\begin{array}{c}\text { IgA } \\
\text { Cut off: } 0.381 \text { OD }\end{array}$} \\
\hline & & & & $\begin{array}{c}\mathrm{OD} \\
\text { index/cutoff }\end{array}$ & Result & $\begin{array}{c}\text { OD } \\
\text { index/cutoff }\end{array}$ & Result & $\begin{array}{c}\text { OD } \\
\text { index/cutoff }\end{array}$ & Result & $\begin{array}{c}\text { OD } \\
\text { index/cutoff }\end{array}$ & Result \\
\hline & & $\begin{array}{l}\text { Vaccine } \\
\text { Dose }\end{array}$ & Age & $0.90-1.10$ & Nesuit & $0.90-1.10$ & Nescuit & $0.90-1.10$ & Nescit & $0.90-1.10$ & Kesut \\
\hline LT & \multirow{3}{*}{$0^{\lambda}$} & 0 & \multirow{3}{*}{60} & 0.08 & - & 0.00 & - & 0.29 & - & 0.03 & - \\
\hline LT & & I & & 0.93 & $+/-$ & 0.70 & - & 0.38 & - & 0.03 & - \\
\hline LT & & II & & 2.63 & + & 1.09 & $+/-$ & 0.46 & - & 0.11 & - \\
\hline $\mathrm{ARB}$ & \multirow{2}{*}{ q } & I & \multirow{2}{*}{61} & 3.16 & + & 0.10 & - & 0.42 & - & 0.00 & - \\
\hline $\mathrm{ARB}$ & & II & & 2.45 & + & 2.49 & + & 1.23 & + & 0.09 & - \\
\hline $\mathrm{VD}$ & \multirow{2}{*}{ q } & I & \multirow{2}{*}{38} & 0.87 & - & 0.42 & - & 0.44 & - & 0.74 & - \\
\hline $\mathrm{VD}$ & & II & & 3.13 & + & 1.17 & + & 0.37 & - & 0.62 & - \\
\hline IDP & \multirow{2}{*}{ q } & I & \multirow{2}{*}{40} & 1.92 & + & 0.23 & - & 0.59 & - & 0.14 & - \\
\hline IDP & & II & & 2.53 & + & 0.88 & - & 0.57 & - & 0.09 & - \\
\hline $\mathrm{PM}$ & \multirow{2}{*}{ q } & I & \multirow{2}{*}{52} & 1.71 & + & 0.09 & - & 0.21 & - & 0.07 & - \\
\hline $\mathrm{PM}$ & & II & & 3.11 & + & 0.31 & - & 0.22 & - & 0.06 & - \\
\hline $\mathrm{AM}$ & \multirow{3}{*}{$\hat{0}$} & 0 & \multirow{3}{*}{59} & 0.30 & - & 0.05 & - & 1.36 & + & 0.04 & - \\
\hline $\mathrm{AM}$ & & I & & 2.84 & + & 0.19 & - & 1.04 & $+/-$ & 0.18 & - \\
\hline $\mathrm{AM}$ & & II & & 2.71 & + & 0.09 & - & 0.79 & - & 0.02 & - \\
\hline $\mathrm{AC}$ & q & II & 58 & 2.77 & + & 0.48 & - & 0.10 & - & 0.02 & - \\
\hline $\mathrm{ACP}$ & q & II & 28 & 2.29 & + & 0.41 & - & 0.40 & - & 0.03 & - \\
\hline MR & \multirow{2}{*}{ q } & I & 56 & 3.01 & + & 1.70 & + & 0.17 & - & 0.08 & - \\
\hline MR & & II & 56 & 2.27 & + & 2.30 & + & 0.13 & - & 0.16 & - \\
\hline MT & 0 & I & 56 & 0.26 & - & 0.23 & - & 0.67 & - & 0.09 & - \\
\hline MT & t & II & 56 & 3.49 & + & 0.36 & - & 0.87 & - & 0.07 & - \\
\hline MLV & 0 & I & 38 & 3.48 & + & 1.94 & + & 0.33 & - & 0.13 & - \\
\hline MLV & 7 & II & 38 & 2.51 & + & 1.13 & + & 0.08 & - & 0.01 & - \\
\hline $\mathrm{RR}$ & $\lambda$ & I & 35 & 1.09 & $+1-$ & 0.10 & - & 0.09 & - & 0.00 & - \\
\hline $\mathrm{RR}$ & 0 & II & 55 & 2.77 & + & 0.63 & - & 0.15 & - & 0.04 & - \\
\hline GDA & $\pi$ & I & 69 & 0.32 & - & 0.00 & - & 0.26 & - & 0.03 & - \\
\hline GDA & 0 & II & (3) & 2.88 & + & 0.61 & - & 0.23 & - & 0.02 & - \\
\hline BR & $\lambda$ & I & 42 & 0.83 & - & 0.09 & - & 0.23 & - & 0.06 & - \\
\hline $\mathrm{BR}$ & 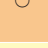 & II & T2 & 2.53 & + & 0.72 & - & 0.31 & - & 0.05 & - \\
\hline $\mathrm{CP}$ & 0 & I & 53 & 2.53 & + & 0.29 & - & 0.49 & - & 0.13 & - \\
\hline $\mathrm{CP}$ & f & II & 30 & 2.87 & + & 0.28 & - & 0.11 & - & 0.04 & - \\
\hline MG & 0 & I & 47 & 0.41 & - & 0.00 & - & 0.17 & - & -0.01 & - \\
\hline MG & 7 & II & 41 & 3.22 & + & 0.37 & - & 0.14 & - & 0.04 & - \\
\hline PT & $\lambda$ & I & 59 & 0.95 & $+/-$ & 0.06 & - & 1.73 & + & 0.04 & - \\
\hline PT & 0 & II & & 2.80 & + & 0.28 & - & 1.53 & + & 0.03 & - \\
\hline $\mathrm{LM}$ & 0 & I & 57 & 0.79 & - & 0.02 & - & 0.27 & - & 0.04 & - \\
\hline LM & t & II & (3) & 2.99 & + & 0.53 & - & 0.30 & - & 0.03 & - \\
\hline DS & 인 & I & 43 & 2.26 & + & 0.20 & - & 1.37 & + & 0.15 & - \\
\hline DS & f & II & 43 & 2.68 & + & 0.02 & - & 0.78 & - & 0.02 & - \\
\hline $\mathrm{MF}$ & 0 & I & 51 & 0.16 & - & -0.04 & - & 0.10 & - & -0.04 & - \\
\hline $\mathrm{MF}$ & 7 & II & $J_{1}$ & 2.70 & + & 0.05 & - & 0.13 & - & 0.01 & - \\
\hline BS & $q$ & II & 31 & 2.87 & + & 0.12 & - & 0.36 & - & 0.05 & - \\
\hline SE & q & II & & 3.03 & + & 0.02 & - & 0.38 & - & 0.02 & - \\
\hline RA & q & II & 59 & 3.96 & + & 0.27 & - & 0.32 & - & 0.02 & - \\
\hline GAM & q & II & 65 & 3.04 & + & 0.17 & - & 0.10 & - & 0.06 & - \\
\hline
\end{tabular}




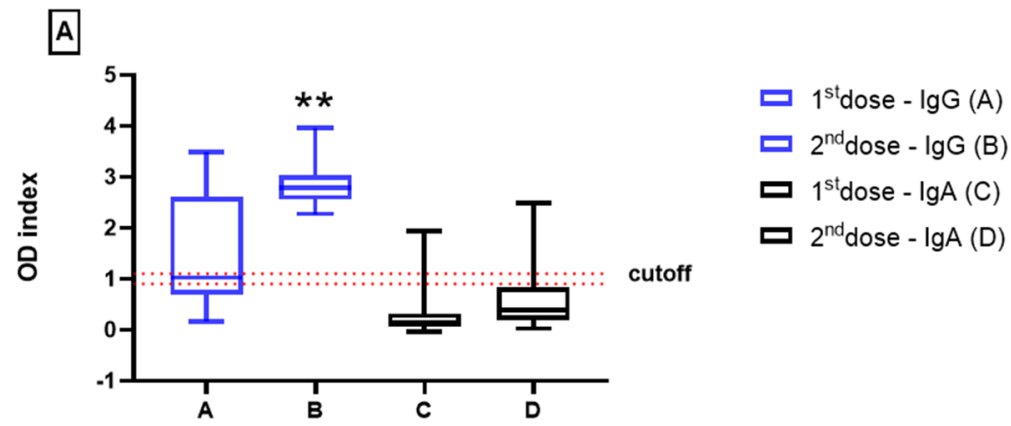

$B$

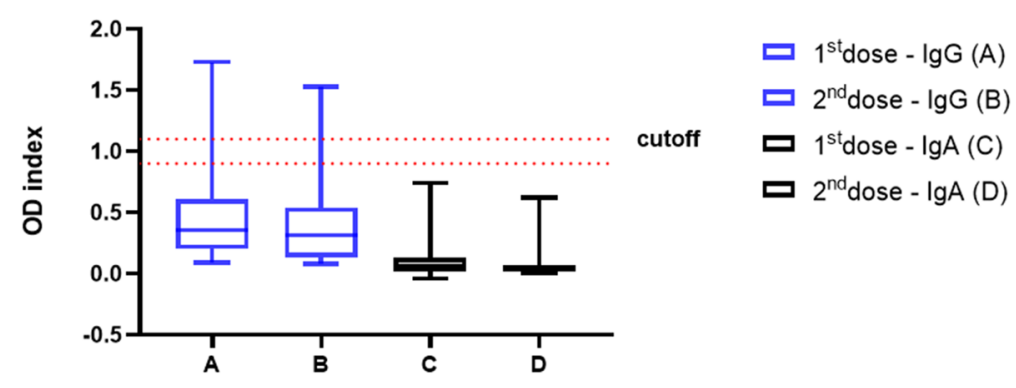

Figure 2. Box \& Whiskers plots show the serological level of $\operatorname{IgG}$ and IgA anti-RBD ectodomain Spike protein (A) and anti-nucleocapsid protein (B) of SARS-CoV-2. Tukey's multiple comparisons test was used (** P value $<0.005)$.

ence of IgG, IgA and also, if desired, IgM antibodies, developed by Bruni et al. (17) proved to be a valuable tool for pre- and post-vaccination antibody monitoring for SARS-CoV-2.

The homemade ELISA test carried out in our laboratory therefore allowed post-vaccine antibody monitoring in 24 healthcare professionals, revealing the full effectiveness of Pfizer vaccine $m R N A B N T 162 b 2$ (Comirnaty) after the second dose. In this way it was also possible to verify the subjective immune response at the first dose, not to mention that it was possible to verify the presence of possible pre-existing anti-Coronavirus antibodies. By evaluating the different antibody levels produced after the vaccination stimulus it would be possible to guess the duration of the immunization and, through monitoring over time, establish when to prepare a possible booster.

Finally, it should be considered that, in the middle of the pandemic period, this type of test, economic, versatile, practical and easy to carry out, would be very useful in developing countries or countries with scarce economic resources.

\section{Acknowledgments}

We thank dr. AR Bruno, dr. MG De Benedittis and mr. A. Masciullo UOS Molecular biology, "S. Caterina Novella" hospital Galatina (LE), for their helpful contribution. We also thank the SOS-Covid team at the European Institute of Oncology, Milan for providing us with antigens and protocols to perform the serological assays.

\section{Funding}

This research received no external funding.

\section{Institutional Review Board Statement}

The study was conducted according to the guidelines of the Declaration of Helsinki and approved by the Ethical Committee of the Lecce Local Health Authority (ASL/LE) (protocol code n. 45 of April 21, 2020).

\section{Informed Consent Statement}

Informed consent was obtained from all subjects involved in the study.

\section{Data Availability Statement}

The data presented in this study are available within the article (Tables 1 and 2 and figures 1 and 2).

\section{Conflicts of Interest}

The authors declare no conflict of interest.

\section{References}

1. Zhou P, Yang X Lou, Wang XG, et al. A pneumonia outbreak associated with a new coronavirus of probable bat origin. Nature. 2020;579(7798):270-273.

2. Zhu N, Zhang D, Wang W, et al. A Novel Coronavirus from Patients with Pneumonia in China, 2019. N Engl J Med. 2020;382(8):727-733.

3. Mahase E. Coronavirus covid-19 has killed more people than SARS and MERS combined, despite lower case fatality rate. BMJ. 2020;368(February):m641. 
4. CRC JHCRC. COVID-19 Dashboard by the Center for Systems Science and Engineering (CSSE) at Johns Hopkins University (JHU).

5. Connors JM, Levy JH. COVID-19 and its implications for thrombosis and anticoagulation. Ann Oncol. 2020;(January):19-21.

6. Gupta A, Malviya A. Chloroquine and hydroxychloroquine for COVID-19: Time to close the chapter. Postgrad Med J. 2020:1-2.

7. Luo E, Zhang D, Luo H, et al. Treatment efficacy analysis of traditional Chinese medicine for novel coronavirus pneumonia (COVID-19): An empirical study from Wuhan, Hubei Province, China. Chinese Med (United Kingdom). 2020;15(1):1-13.

8. Marietta M, Ageno W, Artoni A, et al. COVID-19 and haemostasis: A position paper from Italian Society on Thrombosis and Haemostasis (SISET). Blood Transfus. 2020;18(3):167-169.

9. Pelaia C, Tinello C, Ventrella A, De Sarro G, Pelaia G. Lung under attack by COVID-19-induced cytokine storm: pathogenic mechanisms and therapeutic implications. Ther Adv Respir Dis. 2020;9(6):259-261.

10. Saha A, Ahsan MM, Quader MTU, et al. Clinical characteristics and outcomes of COVID-19 infected diabetic patients admitted in ICUs of the southern region of Bangladesh. Diabetes Metab Syndr Clin Res Rev. 2021;15(1):229-235.

11. Lan J, Ge J, Yu J, et al. Structure of the SARS-CoV-2 spike receptor-binding domain bound to the ACE2 receptor. Nature. 2020;581(7807):215-220.

12. Caini S, Bellerba F, Corso F, et al. Meta-analysis of diagnostic performance of serological tests for SARS-CoV-2 antibodies up to 25 April 2020 and public health implications. Eurosurveillance. 2020;25(23):1-5.

13. To KKW, Tsang OTY, Leung WS, et al. Temporal profiles of viral load in posterior oropharyngeal saliva samples and serum antibody responses during infection by SARSCoV-2: an observational cohort study. Lancet Infect Dis. 2020;20(5):565-574.

14. Kontou PI, Braliou GG, Dimou NL, Nikolopoulos G, Bagos PG. Antibody tests in detecting SARS-CoV-2 infection: a meta-analysis. medRxiv. 2020.

15. Amanat F, Stadlbauer D, Strohmeier S, et al. A serological assay to detect SARS-CoV-2 seroconversion in humans. medRxiv. 2020;2.

16. Seow J, Graham C, Merrick B, et al. Longitudinal evaluation and decline of antibody responses in SARS-CoV-2 infection 2. medRxiv. 2020;(165):1-13.

17. Bruni M, Cecatiello V, Diaz-Basabe A, et al. Persistence of anti-SARS-CoV-2 antibodies in non-hospitalized COVID-19 convalescent health care workers. medRxiv. 2020 . 